\title{
Salivary Matrix Metalloproteinase (MMP)-1 as Non-Invasive Tool for The Diagnosis of Oral Squamous Cell Carcinoma (OSCC)
}

\author{
Sana Jafar Zaidi ${ }^{1}$ \\ Nabeela Riaz ${ }^{2}$ \\ Asifa Iqbal ${ }^{3}$ \\ Ayyaz Ali Khan ${ }^{4}$
}

\author{
BDS, M.Phil \\ BDS, FCPS, MCPS \\ BDS, M.Phil \\ BDS, MSc, PhD
}

OBJECTIVE: To determine the diagnostic accuracy of salivary MMP1 as non-invasive diagnostic biomarker of OSCC through conventional sandwich ELISA technique. Analytical cross-sectional study.

METHODOLOGY: Individuals with clinical suspicion for OSCC (IWCS-OSCC) were included in the study after fulfilling selection criteria. Saliva samples were collected from IWCS-OSCC. To confirm OSCC, the patients were referred for biopsy. After definitive diagnosis on biopsy, patients were labeled OSCC positive or OSCC negative. The colorimetric sandwich-ELISA test was performed on saliva samples to measure the level of MMP1. Data was entered in "Statistical Package for the Social Sciences (SPSS) -16" and levels of MMP1 were correlated with the biopsy status of patient (OSCC positive or OSCC negative). RESULTS: Our sample included twice as many males as females (2.1:1) and a wide age range of 22-77years with median age of 50yrs. Of all the IWCS-OSCC $85 \%$ were OSCC +ve and $15 \%$ were OSCC-ve. Diagnostic accuracy was measured as; Area under curve $(\mathrm{AUC})=0.623$, Sensitivity $(\mathrm{Sn})=50 \%$, Specificity $(\mathrm{Sp})=83.3 \%$, Positive predictive value $(\mathrm{PPV})=94.4 \%$, Negative predictive value $(\mathrm{NPV})=22.7 \%$

CONCLUSION: MMP1 as detected by conventional sandwich-ELISA technique is not proven as an accurate diagnostic biomarker of OSCC.

KEY WORDS: OSCC; MMP1; IWCS-OSCC; Sandwich-ELISA

HOW TO CITE: Zaidi SJ, Riaz N, Iqbal A, Khan AA. Salivary matrix metalloproteinase (MMP)-1 as non-invasive tool for the diagnosis of oral squamous cell carcinoma (OSCC). J Pak Dent Assoc 2021;30(1):18-23.

DOI: https://doi.org/10.25301/JPDA.301.18

Received: 20 July 2020, Accepted: 01 October 2020

\section{INTRODUCTION}

$\mathrm{O}$ SCC is getting global attention for its increasing incidence in youngsters. ${ }^{1}$ On a universal ranking it is the 6th commonest malignancy. ${ }^{2}$ In Pakistan, it is the 2 nd commonest cancer in individuals more than 15 years of age. ${ }^{3}$

In routine, approaches to diagnose OSCC include adequate clinical examination (CE) followed by histological investigation of suspicious sites but they are not efficient to

1. Co-ordinator, Department of Dentistry, Institute of Advanced Dental Sciences and Research (IADSR).

2. Head of Department, Department of Oral \& Maxillofacial Surgery, Mayo Hospital, Lahore.

3. Medical Officer, Department of Oral \& Maxillofacial Surgery, Mayo Hospital, Lahore.

4. Chairperson, Department of Dentistry, Institute of Advanced Dental Sciences and Research (IADSR).

Corresponding author: "Dr. Sana Jafar Zaidi” < sanajaffar98@gmail.com > diagnose OSCC at an earlier stage. ${ }^{4}$ Although, biopsy is considered as gold standard but it is a time consuming ${ }^{5}$ and site specific procedure. ${ }^{6}$ Other issue associated with incisional biopsy is increased chance of metastasis due to breakage in tissue barrier. ${ }^{7}$ In addition to it, high-risk areas with DNA mutation are not always evident on histopathology. So, these factors increase the need of more efficient diagnostic marker to prevent late and missed diagnosis of OSCC. ${ }^{8}$

Salivary detection of diseases has advantage of non-invasiveness, convenience and less discomfortness. These factors make saliva as an efficient medium for the detection of OSCC markers. Nosratzehi observed the levels of MMP1 and other MMPs in saliva of OSCC patients and came to a conclusion that further research is necessary to determine relation between MMPs and OSCC. ${ }^{9}$

MMP1 is a soluble type of interstitial collagenase responsible for the degradation of fibrillar- collagen-I, II and III. ${ }^{9}$ In 2016, salivary MMP1 was introduced in "Taiwan's 
Oral Cancer Screening Program" as a part of non-invasive screening of OSCC. ${ }^{10}$ Stott-miller first time observed high levels of MMP1 proteins in saliva of OSCC patients. ${ }^{11}$

Purpose of this study was to determine diagnostic accuracy of salivary MMP1 as non-invasive diagnostic biomarker of OSCC in Pakistani population by using conventional Colorimetric MMP1 sandwich-ELISA Kit. Saliva was chosen: 1) to identify lesions with suspicion of OSCC at sites difficult to approach for biopsy and 2) to prevent incisional biopsy induced chances of metastasis 3) to avoid complications associated with medically compromised individuals where risks after surgical intervention were high. MMP1 was chosen for its emergence as exceptionally strong biomarker to separate OSCC cases from controls in study carried out by stott-Miller. ${ }^{11}$ In current study Colorimetric MMP1 sandwich ELISA Kit was used to detect MMP1 in Pakistani population with the aim of introducing it in community for screening of OSCC if found accurate for diagnosis.

\section{METHODOLOGY}

In this analytical cross-sectional study, IWCS-OSCC ${ }^{12}$ were selected after ethical approval by IRB, letter No.350/RC/KEMU, from Oral \& Maxillofacial Surgery (OMFS) Department, Mayo Hospital, Lahore after fulfillment of selection criteria during the period of 6 months. Out of one twenty IWCS-OSCC only eighty were included in the study by computer generated random number table, using SPSS-16 software. The patients were then referred to Oral $\&$ Maxillofacial surgeon for biopsy. Histological confirmation via biopsy was considered as "gold standard" internationally for the confirmation of OSCC. ${ }^{6}$ So, the patients showing histopathological findings of OSCC were labelled "OSCC positive" and the patients without histopathological findings of OSCC were labelled "OSCC negative".

Both male and female subjects of age range between 20yrs - 75yrs, visited Oral and Maxillofacial Surgery Department, Mayo Hospital, Lahore and showing clinically suspicion for OSCC, were included in study.

Patients who were not willing to become part of research and showing following conditions with overexpression of MMP1 were excluded from the study.

1- Endometriosis

2- Pyogenic granuloma

3- Pyoderma gangrenosum

4- Decubitus ulcers

5- Stasis ulcers

6- Colorectal adenocarcinoma. ${ }^{13-15}$

Pyogenic granuloma was excluded on the bases of history, $\mathrm{CE}$ and histopathological investigation. Colorectal carcinoma was excluded on the bases of history, CE \& FOBT (sensitivity $>94.1 \%$ and specificity $=87.5 \%) .{ }^{16}$ Endometriosis was excluded in women $\leq 40 \mathrm{yrs}$ of age, on the bases of history of dysmenorrhea (NPV=84.2\%) but all the women with age $>40 \mathrm{yrs}$ were included in the study because of very low prevalence of endometriosis after $40 .{ }^{17,18}$

After informed consent, demographic data, as mentioned in "results" section of article, is collected from patients.

After informed consent, patients were advised not to take food for at least 1 hour before sample collection. Unstimulated saliva was then collected by asking patients to spit into a centrifuge tube. After collection, samples were stored at $4^{\circ} \mathrm{C}$ for 2 hours. Samples were then centrifuged for 10 minutes at $1,300 \mathrm{x} \mathrm{g}$ in centrifuge machine and transferred into cryovials for storage at $-80^{\circ} \mathrm{C} .{ }^{11}$ Sandwich ELISA was performed on saliva samples through MMP1 sandwich-ELISA kit by using microtiter plate washer and reader machines. ${ }^{11,19}$

The ELISA protocol was followed according to the "user guide" provided along with the kit by the trained staff. All stored samples and kits were brought to room temperature before performing the ELISA. All the samples were pre-diluted to 80 folds before performing ELISA.Preparation of standards.

Fifty Microliter of standard diluent was added in each tube. Hundred Microliter of standard (1350 g/L) was Pipetted out in first-tube. From first-tube 1001 was transferred to second-tube. Then 501 was taken out from 2 nd-tube into 3 rd -tube and two more serial dilutions were prepared from 3rd-tube as below in fig-1.

Fig-1: Protocol to Prepare standard solutions

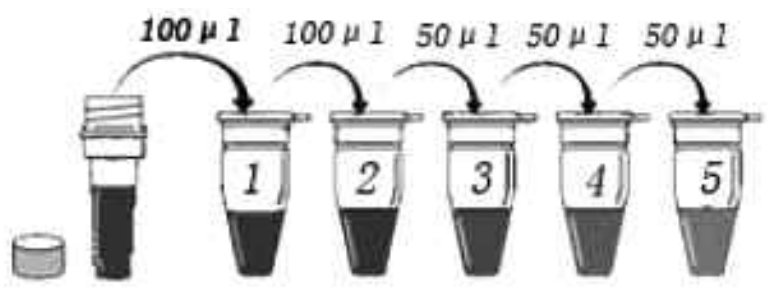

Blank well was set separately (sample \& HRP-Conjugate reagent are not added in blank comparison well but other each step operation is same). Forty Microliter of sample diluent was added to testing sample well; 101 of sample to be tested was added (sample final dilution is 5-fold) and mixed gently. Care was taken not to touch the well wall as far as possible. After covering the wells the plate was incubated at $37^{\circ} \mathrm{C}$ for half an hour.

Thirty-fold diluted wash solution was prepared by diluting wash solution in distilled water. The adhesive strip was uncovered and liquid was discarded. Pipetted washing buffer 
to every well, still for 30 s then drained, repeated 5 times. Then the 501 enzyme, HRP-Conjugate reagent, was added to each well, except in the blank well. The plate was incubated at $37^{\circ} \mathrm{C}$ for half an hour. Again pipetted washing buffer to every well, still for 30s then drained, repeated 5 times. Then 501 of Chromogen Solution A and 501 of Chromogen Solution B was added to each well and light exposure was avoided for 15 minutes at $37^{\circ} \mathrm{C}$. Then the reaction was stopped by adding 501 of "stop solution" to each well (the blue change to yellow).

The plate was placed in microplate reader and absorbance (optical density/ OD) was read at 450nm after pipetting "stop Solution" within $15 \mathrm{~min}$.

"Microsoft office Excel" was used for the calculations of ELISA test. Blank correction was done (OD of the blank well was subtracted from the OD of all wells). Then the OD values and standard concentrations were entered in Excel file. Using "graph" option, standard curve was generated by taking OD values on vertical axis and respective standard concentrations on horizontal axis as shown in fig-2. "Straight line regression" was applied on the standard-curve. By using the Linear-regression equation, $y=m x+b(m=s l o p, x=$ value on $x$-axis, $y=$ value on $y$-axis, $b=$ intercept), "sample concentrations" of MMP1 were calculated after putting their respective OD values in equation.

Fig-2: Standard curve

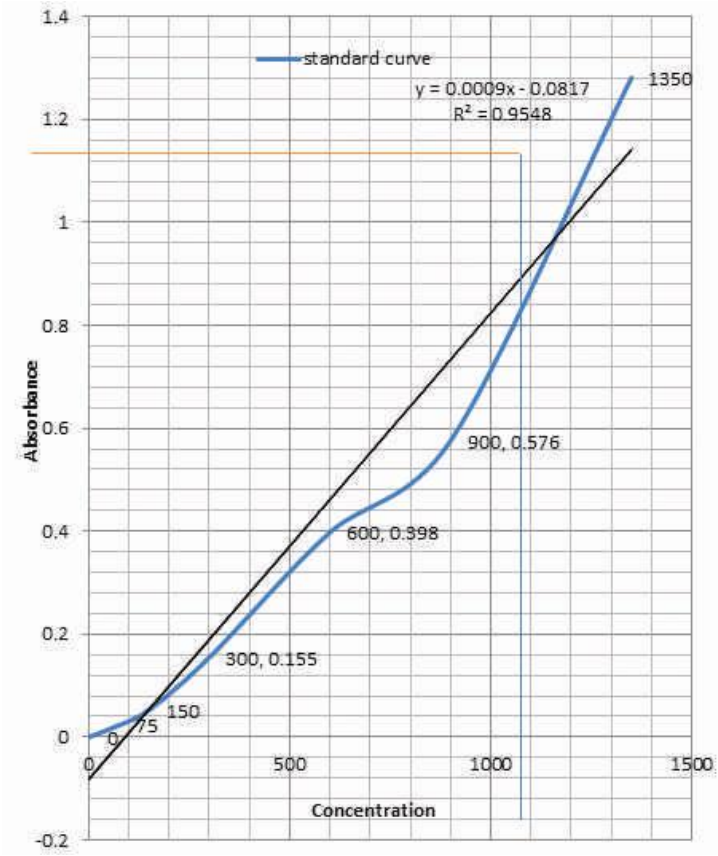

Demographic profile, histopathological findings on biopsy report and, ELISA results after log-transformation using Microsoft Excel were transferred to SPSS-16. Receiver operating characteristic (roc) curve was drawn to measure the cut-off value of MMP1 Concentrations for 80 samples of IWCS-OSCC and it was observed to be $44977.78 \mathrm{~g} / \mathrm{l}$ or $44977780 \mathrm{pg} / \mathrm{ml}$ (log-transformed value $=7.65 \mathrm{pg} / \mathrm{ml})$ as shown in Fig-3. These values of MMP1 concentrations above or equal to cut-off value were labeled as Positive for OSCC and the values below the cut-off value were labelled as Negative for OSCC.

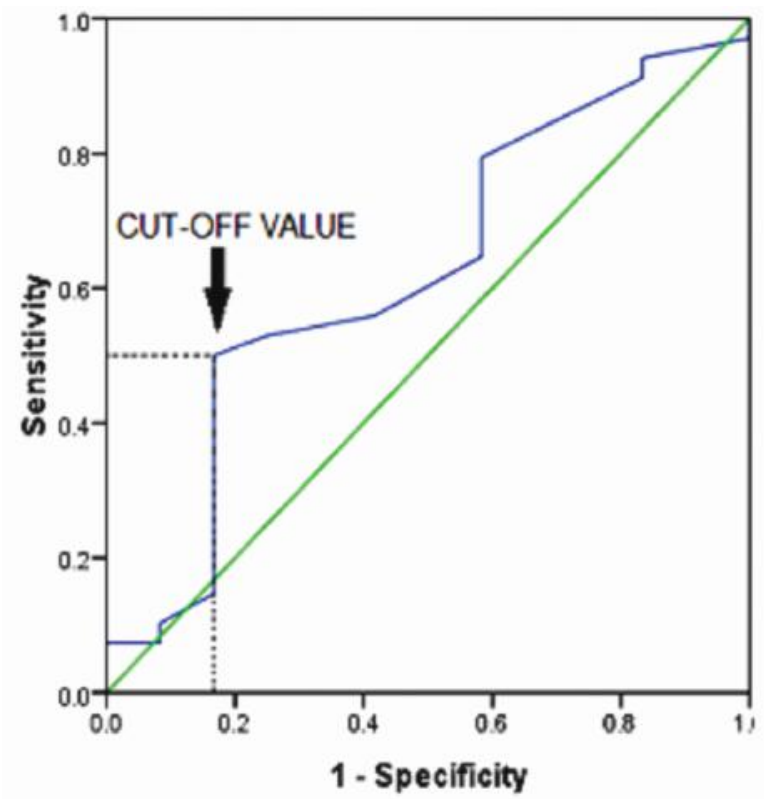

Fig-3: Cutt-off value of Salivary MMp1 for edetection of OSCC

Diagnostic accuracy of salivary MMP1 was measured in terms of AUC (as shown in Fig-4), Sn, Sp, PPV and NPV. Cross-tabulation was generated between histopathological diagnosis (HD) based OSCC +ve and OSCC -ve patients and salivary diagnostic test (SDT) based OSCC +ve and

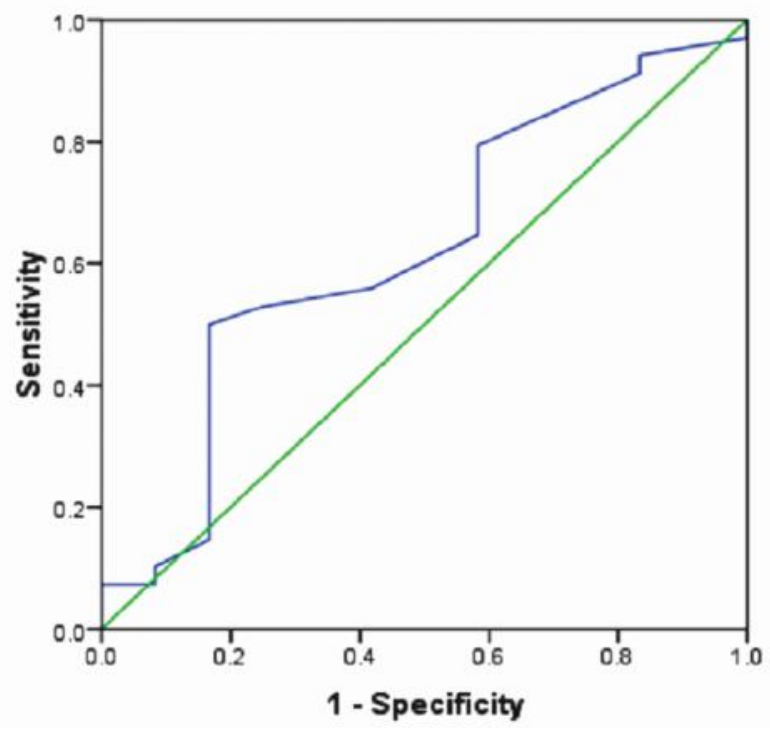

Fig-4: ROC of Salivary MMP-1 
OSCC -ve patients, then the Pearson Chi-Square test was applied to find out $\mathrm{p}$-Value for the association between HD and SDT.

\section{RESULTS}

Of all the IWCS-OSCC, $85 \%$ were OSCC positive and $15 \%$ were OSCC negative upon biopsy. In total $70 \%$ males and $30 \%$ females participated in research. OSCC was found to be more common in males than females with ratio of 2.3:1 Mean age of IWCS-OSCC, OSCC positive patients and OSCC negative patients was $52 \pm 13,53 \pm 13$ yrs and $46 \pm 12$ years respectively. For OSCC positive males, age ranged from $22 \mathrm{yrs}$ to $75 \mathrm{yrs}$.For OSCC positive females, age ranged from $27 \mathrm{yrs}$ to $75 \mathrm{yrs}$. Buccal mucosa was the most common site in our study while exophytic growth as the most common pattern. Most of the patients presented with stage IVa (62.2\%) and WD grade $(76.5 \%)$.

The observed AUC value indicates the poor diagnostic accuracy of salivary MMP1 levels in detection of OSCC as shown in table-1.

Table 1: AUC for salivary mmp1 concentration as diagnostic marker

\begin{tabular}{|c|c|}
\hline \multicolumn{2}{|c|}{ SALIVARY MMP-1 } \\
\hline AUC(S.E) & Significance $(95 \%$ Cl) \\
\hline $0.623(0.089)$ & $\mathrm{P}=0.176(0.449-0.798)$ \\
\hline
\end{tabular}

Relationship between HD and SDT was found to be statistically significant, because $p$-value $=0.032$ is $<0.05$, as shown in table-2.

Table 2: cross-tabulation between histopathological diagnosis and salivary diagnostic test for OSCC.

\begin{tabular}{|c|c|c|c|c|c|}
\hline & \multicolumn{2}{|c|}{$\begin{array}{c}\text { Salivary Diagnostic test for OSCC } \\
\text { (SDT) }\end{array}$} & \multirow[b]{2}{*}{ Total } & \multirow{2}{*}{$\begin{array}{l}\text { P-value of } \\
\text { relationship } \\
\text { b/w HD \& } \\
\text { sDT after } \\
\text { appplying } \\
\text { pearson chi- } \\
\text { square test } \\
\end{array}$} \\
\hline & & $\begin{array}{c}\text { Negative MMP1 } \\
\text { ELISA test }\end{array}$ & $\begin{array}{c}\text { Positve MMP1 } \\
\text { ELISA test }\end{array}$ & & \\
\hline \multirow{2}{*}{$\begin{array}{l}\text { Histopathological } \\
\text { Diagnosis } \\
\text { (HD) }\end{array}$} & SCC & $\begin{array}{l}34 \\
\text { (b) }\end{array}$ & $\begin{array}{l}34 \\
\text { (a) }\end{array}$ & $\begin{array}{c}68 \\
(a+b)\end{array}$ & \multirow[t]{3}{*}{$0.032^{*}$} \\
\hline & $\begin{array}{l}\text { Non- } \\
\text { SCC }\end{array}$ & $\begin{array}{l}10 \\
\text { (d) }\end{array}$ & $\begin{array}{c}2 \\
\text { (c) }\end{array}$ & $\begin{array}{c}12 \\
(c+d)\end{array}$ & \\
\hline \multicolumn{2}{|l|}{ Total } & $44(b+d)$ & $36(a+c)$ & 80 & \\
\hline
\end{tabular}

*Relationship between HD and SDT was found to be statistically significant, because $\mathrm{p}$-value $=0.032$ was $<0.05$.

\section{DISCUSSION}

We found sensitivity of MMP1-salivary diagnostic test as $50 \%$ \& specificity as $83.3 \%$ for the diagnosis of OSCC. These findings support the study conducted by Lallemant et al. who observed the detectable levels of salivary MMP1 mRNA in only $20 \%$ patients of Head \& Neck Cancer (HNC) \& 29\% cases of OSCC. It was also noticed that 9 out of 10 cases of HNC showing detectable levels of salivary-mRNA, belonged to oral region as well as no any control showed detectable mRNA levels. These findings reveal lower sensitivity of this mRNA marker in detection of OSCC but higher level of specificity upto $100 \% .^{20}$

Stott-Miller et al. in 2011, compared the salivary MMP1 levels of OSCC patients with the controls (while the controls were comprised of patients with non-premalignant or non-malignant lesions). He measured the cut-off value of MMP1 (log transformed) as $7.4 \mathrm{pg} / \mathrm{ml}$ that was slightly lower than the cut-off value calculated by us that (log transformed value) was $7.65 \mathrm{pg} / \mathrm{ml} .{ }^{11} \mathrm{He}$ noted the AUC for MMP1 as 0.845 (95\% CI: 0.76-0.93). This value of AUC marks MMP1 as a good diagnostic indicator of OSCC. ${ }^{11}$ In our study, AUC was found to be 0.623 (95\% Cl: 0.449-0.798) that depicts poor diagnostic accuracy of salivary MMP1 in detection of OSCC. Stott-Miller et al. used Chemiluminescent Sandwich-ELISA technique (SearchLight ${ }^{\circledR}$ multiplex sandwich-ELISA proteome array) for the quantification of salivary MMP1 while we used colorimetric ELISA technique for this purpose. Samineni et al. and Hatch et al. found Chemiluminescent technique of Sandwich ELISA as a more sensitive approach than the colorimetric Sandwich-ELISA technique. ${ }^{21}$ So the main reason for this variation in results might be the difference in ELISA technique used for the quantitative analysis of MMP1.

In 2020, Chang et al verified the role of salivary MMP1 in OSCC by using the new in-house technique of ELISA and found MMP1 as an efficient marker to separate OSCC patients from Non-OSCC patients. According to Chang et al, new MMP1 ELISA might be utilized as the supportive tool to detect and monitor OSCC. ${ }^{22}$ Current study showed significant association between salivary MMP1 and OSCC but the diagnostic accuracy was not adequate to use it as screening tool. One of the reason for this low diagnostic accuracy might be the conventional ELISA technique used to measure the Salivary MMP1.

In 2020, Nosratzehi et al compared means of salivary MMP1 levels in three study groups i.e. OSCC group, Oral lichen-planus group and healthy group and found no significant difference in means of salivary MMP1 levels among three groups ${ }^{9}$ that is contrary to our study showing significant association between MMP1 levels and OSCC.

Current study shows the male-female ratio of OSCC 
Salivary matrix metalloproteinase (MMP)-1 as a tool for diagnosis of oral squamous cell carcinoma as $2.1: 1$ that is higher than the ratio $1.4: 1$ presented by Minhas et al. ${ }^{3}$ This variation in both studies may be the due to the exclusion of females showing dysmenorrhea at age $<40 \mathrm{yrs}$ to avoid inclusion of endometriosis cases in current study. On the other hand, Naseer et al. showed comparatively higher male-female ratio 3.7:1 than that of our study. ${ }^{23}$ In Pakistan where gender discrimination is obvious, females are dependent on males to seek for medical healthcare. Mostly they don't travel alone. So, the approach to seek for oral healthcare is mostly influenced by the decision of male family members to bring them hospital for oral examination. ${ }^{12}$ Remarkably higher male-female ratio in study carried out by Naseer et al. might be the reflection of this fact and so far the reason of difference in findings of both researches.

The age we observed for OSCC patients, ranges between 22-75years. Khan et al. in 2015 found the frequency of OSCC in age-group $\leq 40 \mathrm{yrs}$ as $13.04 \% .{ }^{24}$ We observed the frequency of this cancer in these patients as $19.1 \%$. In our study, the prevalence of OSCC in young patients is found higher when compared with other Khan et al.

Buccal-mucosa was the most common site of OSCC in current study that supports the finding of Mirza et al. showing buccal-mucosa as the most common site of OSCC in Pakistani population ${ }^{25}$ while it was also observed to be the most common site among NON-OSCC patients. Minhas et al. found tongue as the most common site in local-population of Lahore. ${ }^{3}$ The reason of this variation in results may be due to the difference in predisposing factors causing OSCC in different communities.

Two recurrent cases of OSCC were noticed in data and all showed positive SDT. This finding supports the relationship of recurrent cases with +ve SDT but this observation was not statistically significant. So, the result obtained might be by chance or the number of recurrent cases were not sufficient to depict statistically significant results.

Stage, grade, site, clinical pattern, histopathological variant and age groups showed no statistically significant relationship with SDT (+ve or -ve).

\section{CONCLUSION}

As detected by colorimetric sandwich-ELISA kit, MMP1 is not proven as an accurate diagnostic biomarker of OSCC. Sensitivity (50\%) of MMP1 levels is too low to be introduced as the screening tool for the detection of OSCC.

\section{RECOMMENDATIONS}

In future, latest techniques such as "Chemiluminescent sandwich-ELISA technique" or "bead-based sandwich-assay technique" with more accurate measuring potential should be experimented to ensure the levels of salivary MMP1 in patients with or without OSCC. In addition, concomitant use of salivary MMP1 with other markers should be tested to look for any improvement in diagnostic accuracy. Salivary MMP1 levels in different types of cancers should be tested to find out its general or specific association with cancers.

\section{CONFLICT OF INTEREST}

None to declare

\section{REFERENCES}

1. Siddiqui IA, Khan H, Siddiqui R, Hafeez M, Dogar MR, Shahid $\mathrm{W}$, et al. Oral cancer frequency at different sub sites presenting at a tertiary care hospital in karachi pakistan. Glob J Otolaryngol 2017;6:4952.

https://doi.org/10.19080/GJO.2017.06.555687

2. Kumar M, Nanavati R, Modi TG, Dobariya C. Oral cancer: Etiology and risk factors: A review. J Cancer Res Ther. 2016;12:458-63 https://doi.org/10.4103/0973-1482.186696

3. Minhas S, Kashif M, Altaf W, Afzal N, Nagi AH. Concomitantchemoradiotherapy-associated oral lesions in patients with oral squamous-cell carcinoma. Cancer Biol Med. 2017;14:176-82. https://doi.org/10.20892/j.issn.2095-3941.2016.0096

4. Csosz É, Lábiscsák P, Kalló G, Márkus B, Emri M, Szabó A, et al. Proteomics investigation of OSCC-specific salivary biomarkers in a Hungarian population highlights the importance of identification of population-tailored biomarkers. PLoS One. 2017;12:e0177282. https://doi.org/10.1371/journal.pone.0177282

5. Omar E. Current concepts and future of noninvasive procedures for diagnosing oral squamous cell carcinoma-a systematic review. Head Face Med. 2015;11:6.

https://doi.org/10.1186/s13005-015-0063-z

6. Vyas T. Biopsy of Oral Lesion-A Review Article. J Adv Med Dent Scie Res. 2018;6:27-35.

7. Berwal V, Kiran S, Naik VG, Khandeparker RVS, Jain H. A review on oral mucosal biopsies with considerations on type of biopsy according to clinical diagnosis and handling of tissues. J Adv Med Dent Sci Res. 2014;2:102-07.

8. Saxena S, Sankhla B, Sundaragiri KS, Bhargava A. A review of salivary biomarker: a tool for early oral cancer diagnosis. Adv Biomed Res. 2017;6:90.

https://doi.org/10.4103/2277-9175.211801

9. Nosratzehi T, Alijani E, Moodi M. Salivary MMP-1, MMP-2, MMP3 and MMP-13 levels in patients with oral lichen planus and squamous cell carcinoma. Asian Pac J Cancer Prev. 2017;18:1947-51. https://doi.org/10.1186/s13104-020-05135-w 
Salivary matrix metalloproteinase (MMP)-1 as a tool for diagnosis of oral squamous cell carcinoma

10. Yu J-S, Chen Y-T, Chiang W-F, Hsiao Y-C, Chu LJ, See L-C, et al. Saliva protein biomarkers to detect oral squamous cell carcinoma in a high-risk population in Taiwan. Proc Natl Acad Sci U S A. 2016;113:11549-554.

https://doi.org/10.1073/pnas.1612368113

11. Stott-Miller M, Houck JR, Lohavanichbutr P, Méndez E, Upton MP, Futran ND, et al. Tumor and salivary matrix metalloproteinase levels are strong diagnostic markers of oral squamous cell carcinoma. Cancer Epidemiol Biomarkers Prev. 2011;20:2628-36.

https://doi.org/10.1158/1055-9965.EPI-11-0503

12. Basharat S, Shaikh BT, Rashid HU, Rashid M. Health seeking behaviour, delayed presentation and its impact among oral cancer patients in Pakistan: a retrospective qualitative study. BMC Health Serv Res. 2019;19:715.

https://doi.org/10.1186/s12913-019-4521-3

13. Teixeira CR, Bonotto ML, Lima JP, Figueiredo LF, Conrado L, Frasca C. Clinical impact of the immunochemical fecal occult blood test for colorectal cancer screening in Brazil. Ann Gastroenterol. 2017;30:442-45.

https://doi.org/10.20524/aog.2017.0151

14. Jiang X-F, Ding L, Tian Y, Han N, Li Z-Q. Interaction of STAT3 and RelB modulates MMP-1 in colon cancer. Chem Biol Interact. 2018;293:94-9.

https://doi.org/10.1016/j.cbi.2018.07.017

15. Rydlova M, Holubec Jr L, Ludvikova Jr M, Kalfert D, Franeková J, Povysil C, et al. Biological activity and clinical implications of the matrix metalloproteinases. Anticancer Res. 2008;28:1389-97.

16. Levi Z, Rozen P, Hazazi R, Vilkin A, Waked A, Maoz E, et al. A quantitative immunochemical fecal occult blood test for colorectal neoplasia. Ann Intern Med. 2007;146:244-55.

https://doi.org/10.7326/0003-4819-146-4-200702200-00003

17. Marasinghe JP, Senanayake H, Saravanabhava N, Arambepola C, Condous G, Greenwood P. History, pelvic examination findings and mobility of ovaries as a sonographic marker to detect pelvic adhesions with fixed ovaries. J Obstet Gynaecol Res. 2014;40:785-90. https://doi.org/10.1111/jog.12234

18. Tahira T, Niaz A, Khalid A. Prevalence of Endometriosis in Patients with Subfertility. Ann Punjab Med Coll. 2019;13:226-28.

19. Smriti K, Ray M, Chatterjee T, Shenoy R-P, Gadicherla S, Pentapati K-C, et al. Salivary MMP-9 as a Biomarker for the Diagnosis of Oral Potentially Malignant Disorders and Oral Squamous Cell Carcinoma. Asian Pac J Cancer Prev. 2020;21:233-38.

https://doi.org/10.31557/APJCP.2020.21.1.233

20. Lallemant B, Evrard A, Combescure C, Chapuis H, Chambon G, Raynal C, et al. Clinical relevance of nine transcriptional molecular markers for the diagnosis of head and neck squamous cell carcinoma in tissue and saliva rinse. BMC Cancer. 2009;9:370.

https://doi.org/10.1186/1471-2407-9-370

21. Kudryashova A, Mikhailova N, Borisova O. Comparison of colorimetric and chemiluminescent ELISA tests for detection of IgG antibodies to human EPO in the sera of experimental animals. Meditsinskaya Immunologiya. 2018;20:935-42.

https://doi.org/10.15789/1563-0625-2018-6-935-942

22. Chang Y-T, Chu LJ, Liu Y-C, Chen C-J, Wu S-F, Chen C-H, et al. Verification of Saliva Matrix Metalloproteinase-1 as a Strong Diagnostic Marker of Oral Cavity Cancer. Cancers. 2020;12:2273. https://doi.org/10.3390/cancers 12082273

23. Naseer R, Naz I, Mahmood MK. Frequency of delayed diagnosis of oral squamous cell carcinoma in Pakistan. Asian Pac J Cancer Prev. 2016;17:5037-40.

24. Khan A, Khan S, Khitab U. Emerging clinical and histopathological spectrum of oral squamous cell carcinoma. J Khyber Coll Dent. 2015;5:12-5.

25. Mirza D, Raza G, Basit A, Naqvi K, Ahmed S, Abassi ZA. Oral squamous cell carcinoma (OSCC) In Karachi city-A retrospective study. Pak Oral Dent J. 2016;36:391-94. 